\title{
Modular Completeness for Communication Closed Layers *
}

\author{
Maarten Fokkinga, Mannes Poel \& Job Zwiers \\ University of Twente ${ }^{\dagger \ddagger}$
}

\begin{abstract}
The Communication Closed Layers law is shown to be modular complete for a model related to that of Mazurkiewicz. It is shown that in a modular style of program development the CCL rule cannot be derived from simpler ones. Within a non-modular set-up the CCL rule can be derived however from a simpler independence rule and an analog of the expansion rule for process algebras.
\end{abstract}

\section{Introduction}

In an earlier paper [JPZ91] a formulation of the principle of communication closed layers [EF82] by means of an algebraic rule was proposed. This Communication Closed Layers law (CCL) has been applied thereafter in the construction and verification of various algorithms and protocols [JZ92b, JZ92a, JPSZ91, Zwi91], among which are a version of the Two Phase Commit protocol [JZ92b] and a rather complicated minimum weight spanning tree algorithm by Gallager, Humblet and Spira [JZ92a, GHS83, SdR89, CG88]. The versatility of the CCL rule, especially within a modular style of program development, stems from the possibility to rewrite a distributed process into a so-called layered form which can often be analyzed by means of simpler techniques for sequential programs. Parallel composition is denoted $P \| Q$ and layered composition, being a weakened version of sequential composition, is denoted $P \bullet Q$. The use of a partial order model, and exploitation of independence information enable transformations of concurrent systems into more sequential versions without the expense of introducing extra nondeterminism. A similar methodology of program construction underlies work on ISTL (interleaving set temporal logic) [KP87, KP89, KP90, KP92]. The CCL law allows one to prove equalities between processes based on independence information. In general the rule states that whenever $P$ is independent of $S$ and moreover $Q$ is independent of $R$ then the following equality holds:

$$
(P \bullet Q) \|(R \bullet S)=(P \| R) \bullet(Q \| S)
$$

The notion of independence relies on the particular model of concurrency under concern; e.g. for shared variable models $P$ and $S$ are said to be independent whenever they access

\footnotetext{
${ }^{*}$ Part of this work has been supported by Esprit/BRA Project 6021 (REACT).

${ }^{\dagger}$ Department of Computer Science, P.O. Box 217, 7500 AE Enschede, The Netherlands. E-mail: \{fokkinga,mpoel,zwiers\}@cs.utwente.nl

${ }^{\ddagger}$ The authors thank Wil Janssen for carefully reading the manuscript.
} 
disjoint sets of shared variables, whereas for communication based models independence of $P$ and $S$ means that they communicate along disjoint sets of channels.

Note that the CCL law does not rely on the internal structure of $P, Q, R$ and $S$. In fact, $P, Q, R$ and $S$ are best regarded as typed process variables where the typing information allows one to decide whether the processes denoted by variables are independent or not, but anymore detailed information is not available. This is important for a modular style of process derivation, as we will explain now. If $P, Q, R$ and $S$ in the CCL law are instantiated (i.e. substituted) by concrete process terms that are built up from atomic actions, then we show that the law can be derived from simpler axioms among which are an expansion law for parallelism, and the independence axiom:

$$
P \bullet Q=P \| Q=Q \bullet P \text { if } P \text { is independent from } Q \text {. }
$$

Yet, in order to show equality between $(P \bullet Q) \|(R \bullet S)$ and $(P \| R) \bullet(Q \| S)$ based on these simpler laws involves transforming the internal structure of $P, Q, R$ and $S$. For modular reasoning this is exactly what one would like to avoid.

Modular completeness [Zwi89] refers to the following completeness property. Let "P sat spec" denote that process $P$ satisfies some specification spec. (Simple) completeness of a proposed axiomatization means that whenever $P$ sat spec is a valid formula, then it is also (formally) derivable. Now assume that $P\left(X_{0}, \ldots, X_{n}\right)$ is a process term containing free process variables $X_{0}, \ldots, X_{n}$. Modular completeness means that whenever a formula of the form

$$
\operatorname{spec}^{\prime}\left(X_{0}, \ldots, X_{n}\right) \rightarrow \operatorname{spec}\left(P\left(X_{0}, \ldots, X_{n}\right)\right)
$$

is valid, then it is formally derivable within the axiomatization under consideration. For the present context, the specifications on the left hand side (" $\operatorname{spec}^{\prime}\left(X_{0}, \ldots, X_{n}\right)$ ") take on the form of a list of independence relations between process variables, of the form $X_{i} \# X_{j}$. The right hand side has the form of an equality " $P\left(X_{0}, \ldots, X_{n}\right)=Q\left(X_{0}, \ldots, X_{n}\right)$ ".

The main result of this paper states that a few basic axioms together with the CCL law form a modular complete system. Moreover, we argue that such a completeness result is not possible with rules that are any simpler than the CCL rule. For instance, the following simplified versions of the CCL rule do not lead to a complete system:

Provided that $P$ and $S$ are independent and $Q$ and $R$ are independent:

$$
\begin{aligned}
P \| S & =P \bullet S & & \text { (Independence) } \\
(P \bullet Q) \| S & =P \bullet(Q \| S) & & \text { CCL-L) } \\
(P \bullet Q) \| R & =(P \| R) \bullet Q & & (\text { CCL-R })
\end{aligned}
$$

In fact it can be shown that if a system has rules only in the form of equalities between process terms that have less than four variables, the CCL rule is not derivable, and therefore such a system cannot be complete.

The completeness result is with respect to a partial order model of concurrency, related to Mazurkiewicz' trace model [Maz89]. It differs from simple partial order models in that it is based on both an partial order relation and a separate concurrency relation. 
For a comparison we also consider processes that are built up from concrete atomic actions, rather than from typed process variables. Within this setting the CCL law still makes sense as it allows for a modular style of reasoning. But a non-modular style that avoids the CCL rule becomes also a possibility. This can be achieved for instance by introducing an analog of the expansion law for CCS [Mil80] that allows one to replace parallel composition by layered composition and nondeterministic choice. A few basic axioms together with the analog of the expansion law and the simple "Independence" axiom mentioned above turn out to form already a complete axiomatization. Thus the CCL law is seen to be a derived law within this context.

Finally we mention here that when no knowledge concerning independence is taken into account or, equivalently, when we assume that no two process variables are independent, process equality is already axiomatized completely [Gis84] by only a few simple axioms concerning commutativity of $\|$ and associativity of $\|$ and $\bullet$.

\section{Two process languages}

We introduce two process languages that both are tiny subsets of the languages discussed in e.g. [JPZ91, JPZ93, Zwi91]. Their sole purpose is to have a framework to discuss our completeness results in later sections.

Let (Act, $\leadsto$ ) be a given (countable) set of atomic actions $(a \in) \mathcal{A c t}$, together with a symmetric irreflexive dependence relation " w". (Within specific applications, such as database serializability theory, dependency between transactions is also called conflict. The term conflict relation instead of dependence relation is also used in [JPZ91, JPZ93, Zwi91].) In many cases we use the independence relation " $\sharp$ ", which is just the complement of " $\leadsto$ ". An alphabet $\alpha$ is defined here as a subset of Act. Two alphabets $\alpha$ and $\beta$ are independent iff all actions $a \in \alpha$ are independent of all $b \in \beta$. We assume that there is a set $\left(X_{\alpha} \in\right)$ Pvar of process variables, each typed by means of some alphabet $\alpha$. The alphabet $\alpha(P)$ of process terms $P$ is defined as the set of all actions occurring in $P$. Two processes $P$ and $Q$ or two process variables $X_{\alpha}$ and $X_{\beta}$ are said to be independent iff their associated alphabets are independent. This is denoted by $P \sharp Q$ (i.e. not $P \leadsto Q$ ) and $X_{\alpha} \sharp X_{\beta}$, respectively. The two languages $L_{\text {pvar }}$ and $L_{a t o m i c}$ are defined thus:

$$
\begin{aligned}
& P \in L_{\text {pvar }}, \\
& P::=X_{\alpha}|\quad P \bullet Q| \quad P \| Q \mid \text { skip } \\
& P \in L_{\text {atomic }}, \\
& P::=a|\quad P \bullet Q| \quad P \| Q \mid \quad P \text { or } Q \quad \mid \text { skip | empty }
\end{aligned}
$$

A partial order model for $L_{\text {atomic }}$ is given below in section 3.3. Here we provide some intuition.

A basic assumption is that a (single) computation or run of a system can be modeled as a partially ordered multiset (pomset) of actions. (The usual distinction between actions and events, i.e., instances of actions, need not be made here). Actions within a given run remain unordered iff they are independent. Two processes $P$ and $Q$ are regarded as equal, denoted by $P=Q$, iff their sets of pomsets are equal. 
Parallel composition $P \| Q$ executes $P$ and $Q$ with dependent actions of $P$ and $Q$ ordered nondeterministically. Thus $P \| Q$ denotes a set of runs. The nondeterministic choices for different pairs of dependent actions are of course subject to the condition that the order must be acyclic.

For layer composition $P \bullet Q$ the situation is somewhat like parallel composition, the difference being that when a $P$ action $a$ and a $Q$ action $b$ are dependent then the $a$ can only precede $b$. (For parallel composition $b$ could also precede $a$.) Layer composition should be distinguished from classical sequential composition $P$; $Q$, which would require that all $P$ actions precede all $Q$ actions, irrespective of the dependence relation. One could view $P ; Q$ as a degenerate case where the independence relation is empty, i.e. no two actions are independent.

The process skip performs no action and acts as the unit element for layer and parallel composition.

Nondeterministic choice $P$ or $Q$ is a straightforward construct that executes either $P$ or $Q$. The related process empty acts as the unit for the choice construct and as "zero" for parallel and layer composition. We use the abbreviation $\Sigma_{i \in I} P_{i}$ for choices of the form $P_{i_{0}}$ or $P_{i_{1}}$ or $\cdots$, where $i_{j} \in I$.

\section{Axiomatization}

In this section we present some groups of axioms for the two process languages $L_{p v a r}$ and $L_{\text {atomic }}$ and give sound models for both.

\subsection{Axioms for $L_{\text {pvar }}$}

Axioms A1

$$
\begin{aligned}
& P\|Q=Q\| P \quad(\mathrm{COM} 1) \\
& P\|(Q \| R)=(P \| Q)\| R \text { (ASSOC1) } \\
& P \bullet(Q \bullet R)=(P \bullet Q) \bullet R \quad(\text { ASSOC2) } \\
& P \| \text { skip }=P \quad \text { (SKIP1) } \\
& P \text {. skip }=P \quad \text { (SKIP2) } \\
& \text { skip } \bullet=P \quad \text { (SKIP3) }
\end{aligned}
$$

Axioms A2

Provided that $P \sharp S$, and $Q \sharp R$ :

$$
(P \bullet Q) \|(R \bullet S)=(P \| R) \bullet(Q \| S) \quad(\mathrm{CCL})
$$

\section{Remark.}

From the Communication Closed Layers law (CCL) and the axioms for skip from A1, the following laws can be derived:

Provided that $P \sharp S$, and $Q \sharp R$ :

$$
\begin{array}{rlll}
(P \bullet Q) \| S & =P \bullet(Q \| S) & & (\mathrm{CCL}-\mathrm{L}) \\
(P \bullet Q) \| R & =(P \| R) \bullet Q & & (\mathrm{CCL}-\mathrm{R}) \\
P \| S & =P \bullet S & & \text { (Independence) }
\end{array}
$$

(End of remark) 


\subsection{Axioms for $L_{\text {atomic }}$}

Axioms A3

$$
\begin{aligned}
& P \text { or } Q=Q \text { or } P \\
& P \text { or }(Q \text { or } R)=(P \text { or } Q) \text { or } R \\
& P \text { or } P=P \\
& P \|(Q \text { or } R)=(P \| Q) \text { or }(P \| R) \\
& P \bullet(Q \text { or } R)=(P \bullet Q) \text { or }(P \bullet R) \\
& (P \text { or } Q) \bullet R=(P \bullet R) \text { or }(Q \bullet R) \\
& P \text { or empty }=P \\
& P \| \text { empty }=\text { empty } \\
& P \cdot \text { empty }=\text { empty } \\
& \text { empty } \bullet P=\text { empty }
\end{aligned}
$$

In order to formulate the expansion law for $L_{\text {atomic }}$ we introduce the notion of (syntactic) traces:

Definition 3.1 Syntactic Traces

A (syntactic) trace is a process term of the form $a_{1} \bullet a_{2} \bullet \cdots \bullet a_{n}$ where all the $a_{i}$ are atomic actions. The case $n=0$ is allowed, and is identified with the skip process.

Definition 3.2 Minimal Elements

Let $t=t_{1} \bullet \cdots \bullet t_{n}$ be a trace. The set of minimal elements of $t$ is:

$$
\operatorname{Minimal}(t) \stackrel{\text { def }}{=}\left\{t_{i} \mid \forall j<i, t_{j} \sharp t_{i}\right\}
$$

Let $t^{(i)}$ denote the trace $t$ with $t_{i}$ removed, i.e.

$$
t^{(i)}=t_{1} \bullet \cdots \bullet t_{i-1} \bullet t_{i+1} \bullet \cdots \bullet t_{n} \quad(1 \leq i \leq n)
$$

\section{Axioms A4}

Let $P=p_{1} \bullet p_{2} \bullet \cdots \bullet p_{n}$ and $Q=q_{1} \bullet q_{2} \bullet \cdots \bullet q_{m}$ be (syntactic) traces, where $n, m \geq 1$.

$$
P \| Q=\Sigma_{p_{i} \in \operatorname{Minimal}(P)} p_{i} \bullet\left(P^{(i)} \| Q\right)
$$

or

$$
\Sigma_{q_{i} \in \operatorname{Minimal}(Q)} q_{i} \bullet\left(P \| Q^{(i)}\right)
$$

Provided that for actions $a$ and $b, a \sharp b$

$$
a \| b=b \bullet a \quad \text { (Act-Independence) }
$$

\section{Remark.}

- Laws like the Independence axiom for processes (rather than for actions), or the CCL laws are all valid for the model for $L_{\text {atomic }}$ that we discuss below. Here we have included the minimum for the completeness result.

- Note that for the degenerated case where we have an empty independence relation, the above expansion law boils down to an expansion law for interleaving semantics. For in that case, only $p_{1}$ and $q_{1}$ are minimal elements.

- If $a$ and $b$ are independent actions, i.e. $a \sharp b$, then such actions can be interchanged:

$$
a \bullet b=b \bullet a
$$


- The expansion axiom presented here is a slight simplification of a similar law in [PZ92].

(End of remark)

\subsection{The Partial Order Model for $L_{\text {atomic }}$}

We construct a model for the language $L_{\text {atomic }}$ for which all of the axioms (A1), (A2), (A3) and (A4) are valid. It is shown in the corresponding report, [PZ93b], that axioms (A1), (A3) and (A4) form a complete system with respect to this model.

A partially ordered action set is defined as a structure $(E, \longrightarrow)$ where $E$ is a multiset of actions, and where $\rightarrow$ is an irreflexive partial order on $E$. A partially ordered action multiset $(E, \longrightarrow)$ is conflict closed if for any two $a_{0}, a_{1} \in E$ it is the case that they are ordered if they are dependent:

$$
a_{0} \longrightarrow a_{1} \text { or } a_{1} \longrightarrow a_{0} \text { if } a_{0} \leadsto a_{1} .
$$

A run or computation is defined to be a partially ordered action set that is conflict closed. The set of all such runs is denoted by $\mathcal{P} O_{0}$.

Definition 3.3 Semantic operators

Let as usual $R^{+}$denote the transitive closure of binary relation $R$.

For runs $H_{0}=\left(E_{0}, \longrightarrow_{0}\right)$ and $H_{1}=\left(E_{1}, \longrightarrow_{1}\right)$ in $\mathcal{P} O_{0}$ define

- the parallel composition by

$$
\begin{aligned}
& H_{0} \| H_{1}=\left\{H \in \mathcal{P} O_{0} \quad \mid \quad H=\left(E_{0} \cup E_{1},\left(\rightarrow_{0} \cup \rightarrow_{1} \cup \rightarrow_{C}\right)^{+}\right)\right. \text {, where } \\
& \quad \rightarrow_{C} \subseteq\left(\left(E_{0} \times E_{1}\right) \cup\left(E_{1} \times E_{0}\right)\right) \text { such that } \\
& \left.\quad\left(\left(e, e^{\prime}\right) \in \rightarrow_{C} \vee\left(e^{\prime}, e\right) \in \rightarrow_{C}\right) \text { iff } e \leadsto m e^{\prime}\right\}
\end{aligned}
$$

- and layer composition by

$$
\begin{gathered}
H_{0} \bullet H_{1}=\left\{H \in \mathcal{P} O_{0} \quad \mid \quad H=\left(E_{0} \cup E_{1},\left(\rightarrow_{0} \cup \rightarrow_{1} \cup \rightarrow_{C}\right)^{+}\right)\right. \text {, where } \\
\left.\rightarrow_{C} \subseteq\left(E_{0} \times E_{1}\right) \text { such that }\left(\left(e, e^{\prime}\right) \in \rightarrow_{C}\right) \text { iff } e \leftrightarrow e^{\prime}\right\}
\end{gathered}
$$

Observe that $H_{0} \bullet H_{1}$ consists of a single run.

All unions in the above definition should be understood as disjoint unions. Note that the only difference between parallel and layered composition is in the restrictions on $\rightarrow_{C}$, hence $H_{0} \bullet H_{1} \subseteq H_{0} \| H_{1}$. We define the semantics of the language $L_{\text {atomic }}$ by means of a semantic meaning function of the form

$$
\llbracket \cdot \rrbracket_{\text {at }}: L_{\text {atomic }} \rightarrow \mathcal{P}\left(\mathcal{P O} O_{0}\right) .
$$

Definition 3.4 Semantics of $L_{\text {atomic }}$

$$
\begin{aligned}
& \llbracket a \rrbracket_{a t}=\{(a, \emptyset)\} \\
& \llbracket P \| Q \rrbracket_{a t}=\bigcup\left\{H_{P} \| H_{Q} \mid H_{P} \in \llbracket P \rrbracket_{a t}, H_{Q} \in \llbracket Q \rrbracket_{a t}\right\}
\end{aligned}
$$




$$
\begin{aligned}
& \llbracket P \cdot Q \rrbracket_{a t}=\bigcup\left\{H_{P} \bullet H_{Q} \mid H_{P} \in \llbracket P \rrbracket_{a t}, H_{Q} \in \llbracket Q \rrbracket_{a t}\right\} \\
& \llbracket P \text { or } Q \rrbracket_{a t}=\llbracket P \rrbracket_{a t} \cup \llbracket Q \rrbracket_{a t} \\
& \llbracket \text { skip } \rrbracket_{a t}=\{(\emptyset, \emptyset)\} \\
& \llbracket \text { empty } \rrbracket_{a t}=\emptyset
\end{aligned}
$$

\section{Theorem 3.5 Soundness}

The axioms (A1), (A2), (A3) and (A4) are sound with respect to the partial order model defined above.

The proof is omitted. Soundness of these axioms for a related (more complicated) model can be found in an earlier paper, [JPZ93].

\section{Theorem 3.6 Completeness}

The axioms (A1), (A3), and (A4) form a complete system for $L_{\text {atomic }}$.

A proof of the completeness theorem can be found in [PZ93b]. It is shown in [JPZ91, PZ92, PZ93a] that the CCL law holds for (a slightly more complicated model than) $L_{\text {atomic }}$. Hence

\section{Corollary 3.7}

The CCL law is a derived law in the model $L_{\text {atomic }}$

\subsection{The Graph Model for $L_{p v a r}$}

We present a more complicated partial order model for the $L_{p v a r}$ language. Runs in the form of pomsets of actions don't suffice here, as the basic terms in $L_{\text {pvar }}$ are variables, not actions. Rather we use runs that take on the form of graphs $G=(V, \rightarrow,-)$ with two kind of arcs, namely the concurrency arc - and the precedence arc $\rightarrow$. Formally, $\rightarrow$ is an irreflexive partial order on the set $V$ of vertices, whereas the - is just a symmetric binary relation on $V$. The precedence relation $\rightarrow$ has the same role as in the simple model of the previous section. We shall now explain the reason for introducing the concurrency relation

The intended meaning of $X_{\alpha} \rightarrow Y_{\beta}$ and $X_{\alpha}-Y_{\beta}$ is as follows. Let $a, b$ be dependent actions. Then after refining the variables into concrete processes with action $a$ occurring in $X_{\alpha}$ and $b$ in $Y_{\beta}$, we shall have $a \rightarrow b$ in case of $X_{\alpha} \rightarrow Y_{\beta}$, but either $a \rightarrow b$ or $b \rightarrow a$ in case of $X_{\alpha}-Y_{\beta}$. So, if both $a, a^{\prime}$ eventually occur in place of $X_{\alpha}$, and $b, b^{\prime}$ in place of $Y_{\beta}$, then in the case of $X_{\alpha}-Y_{\beta}$ we may have $a \rightarrow b$ and $b^{\prime} \rightarrow a^{\prime}$ at the same time. This is not expressible by either $X_{\alpha} \rightarrow Y_{\beta}$ nor $Y_{\beta} \rightarrow X_{\alpha}$.

We will now give the semantics for $L_{\text {pvar }}$ terms. $P$. Informally the semantics $\llbracket P \rrbracket_{[} v a r$ is the graph where the vertices $V$ is the set of variables occurring in $P$. The binary relation $\rightarrow$ on $V$ is defined as follows: $X_{\alpha} \rightarrow Y_{\beta}$ if and only if $X_{\alpha} \leadsto Y_{\beta}$ and there is a syntactic subexpression $P_{1} \bullet P_{2}$ of $P$ such that $X_{\alpha}$ is a variable of $P_{1}$ and $Y_{\beta}$ is a variable of $P_{2}$. Similarly the symmetric binary relation - on $V$ is defined as: $X_{\alpha}-Y_{\beta}$ if and only if $X_{\alpha} \leadsto Y_{\beta}$ and there is a syntactic subexpression $P_{1} \| P_{2}$ of $P$ such that $X_{\alpha}$ is a variable of $P_{1}$ and $Y_{\beta}$ is a variable of $P_{2}$, or $X_{\alpha}$ in $P_{2}$ and $Y_{\beta}$ in $P_{1}$.

Formally the semantics is given as follows. For two graphs $G_{Q}$ and $G_{P}$ define

$$
G_{Q} \| G_{R} \stackrel{\text { def }}{=}\left(V_{Q} \cup V_{R}, \rightarrow_{Q} \cup \rightarrow_{R},-{ }_{Q} \cup-{ }_{R} \cup-\|\right)
$$


where

$$
\text { - } \stackrel{\text { def }}{=}\left\{(S, T) \mid\left(\left(S \in V_{Q} \wedge T \in V_{R}\right) \vee\left(S \in V_{R} \wedge T \in V_{Q}\right)\right) \wedge S \leftrightarrow T\right\}
$$

and

$$
G_{Q} \bullet G_{R} \stackrel{\text { def }}{=}\left(V_{Q} \cup V_{R}, \rightarrow_{Q} \cup \rightarrow_{R} \cup \rightarrow_{\bullet},-{ }_{Q} \cup-{ }_{R}\right)
$$

where

$$
\rightarrow \bullet \stackrel{\text { def }}{=}\left\{(S, T) \mid\left(S \in V_{Q} \wedge T \in V_{R}\right) \wedge S \text { mm } T\right\}
$$

Then

$$
\begin{aligned}
& \llbracket X_{\alpha} \rrbracket_{v a r}=\left(X_{\alpha}, \emptyset, \emptyset\right) \\
& \llbracket P\left\|Q \rrbracket_{v a r}=\llbracket P \rrbracket_{v a r}\right\| \llbracket Q \rrbracket_{v a r} \\
& \llbracket P \cdot Q \rrbracket_{v a r}=\llbracket P \rrbracket_{v a r} \bullet \llbracket Q \rrbracket_{v a r} \\
& \llbracket \text { skip } \rrbracket_{v a r}=(\emptyset, \emptyset, \emptyset)
\end{aligned}
$$

\subsubsection{Relation between the partial order model and the graph model}

Let $P \in L_{\text {atomic }}$ with alphabet $\alpha$ and $Q \in L_{\text {atomic }}$ with alphabet $\beta$, and let $H_{P} \stackrel{\text { def }}{=}\left(E_{P}, \rightarrow_{P}\right) \in$ $\llbracket P \rrbracket_{a t}$ and $H_{Q} \stackrel{\text { def }}{=}\left(E_{Q}, \rightarrow_{Q}\right) \in \llbracket Q \rrbracket_{a t}$ Observe that $H_{P}$ and $H_{Q}$ can be seen as graphs in the above sense, with empty - relation. Thus consider $H_{P}$ and $H_{Q}$ as graphs, say $\mathcal{H}_{P}$ and $\mathcal{H}_{Q}$ respectively. Then $\mathcal{H}_{P} \| \mathcal{H}_{Q}$ is the graph $\left(E_{P} \cup E_{Q}, \rightarrow_{P} \cup \rightarrow_{Q},-_{P Q}\right)$ where the set of vertices $E_{P} \cup E_{Q}$ consists of atomic actions, and for each pair of atomic actions $a, b$

$$
(a, b) \in-_{P Q} \text { iff }(a \leftrightarrow b) \wedge\left(a \in E_{P} \leftrightarrow b \in E_{Q}\right)
$$

In other words, $(a, b) \in-{ }_{P Q}$ if they are dependent and one them is contained in $E_{P}$ and the other in $E_{Q}$. The intuitive meaning of $a-b$ is that in actual run $a$ must precede $b$, i.e. $a \rightarrow b$, or conversely $b$ must precede $a$, i.e. $b \rightarrow a$. Thus in order to transform the graph into an actual run one must make for each pair of atomic actions $a, b$ with $\{(a, b),(b, a)\} \subseteq-P Q$ a choice, namely $a \rightarrow b$ or $b \rightarrow a$. If one transforms each $a-b$ into $a \rightarrow b$ or $b \rightarrow$ one gets a graph of the form.

$$
\left(E_{P} \cup E_{Q}, \rightarrow_{P} \cup \rightarrow_{Q} \cup \rightarrow_{C}, \emptyset\right)
$$

where

$$
\rightarrow_{C} \subseteq\left(E_{P} \times E_{Q}\right) \cup\left(E_{Q} \times E_{P}\right) \text { such that }\left((a, b) \in \rightarrow_{C} \vee(b, a) \in \rightarrow_{C}\right) \text { iff } a \leadsto b
$$

Observe that the above formula closely resembles the definition of parallel composition in Definition 3.3. If $\rightarrow_{P} \cup \rightarrow_{Q} \cup \rightarrow_{C}$ is acyclic then

$$
\left(E_{P} \cup E_{Q},\left(\rightarrow_{P} \cup \rightarrow_{Q} \cup \rightarrow_{C}\right)^{+}\right)
$$

is a run of $H_{P} \| H_{Q}$. In the above construction there was choice, namely transform $a-b$ to $a \rightarrow b$ or $b \rightarrow a$. If one takes the union over all such choices the above procedure will generate exactly $H_{P} \| H_{Q}$.

Hence if one applies the above transformation to all $H_{P} \in \llbracket P \rrbracket_{a t}$, and all $H_{Q} \in \llbracket Q \rrbracket_{a t}$ one precisely obtains all runs in $\llbracket P \| Q \rrbracket_{a t}$.

For $\mathcal{H}_{P} \bullet \mathcal{H}_{Q}$ the situation is not so complex

$$
\mathcal{H}_{P} \bullet \mathcal{H}_{Q}=\left(E_{P} \cup E_{Q}, \rightarrow_{P} \cup \rightarrow_{Q} \cup \rightarrow_{C}, \emptyset\right)
$$


Figure 1: The graph of both $(P \bullet Q) \|(R \bullet S)$ and $(P \| R) \bullet(Q \| S)$

that $P, Q, R$ and $S$ are arbitrary process terms, not just variables. In that case the graph in figure 1 can be obtained as follows. First compute the graphs of $P, Q, R$ and $S$. Then connect each vertex of $P$ with - to each dependent vertex of $R$. Similarly for $Q$ and $S$. Finally connect each vertex of $P$ with a $\rightarrow$ to each dependent vertex of $Q$. Similarly for $R$ and $S$. From this construction it is seen that the graphs for $(P \bullet Q) \|(R \bullet S)$ and $(P \| R) \bullet(Q \| S)$ are, again, the same.

The next section is dedicated to the proof of

Theorem 3.9 Modular Completeness

The axioms (A1) and (A2) form a complete system for $L_{\text {pvar }}$

\subsection{Completeness of the CCL Rule}

The aim of this section is to prove that the axioms (A1) together with (A2) (the CCL law) are modular complete. More precisely we will show that when two syntactic terms $P$ and $P^{\prime}$ represent the same graph $\mathcal{G}$ in the graph model, $P$ can be transformed into $P^{\prime}$, using only the axioms (A1) and (A2). 
Figure 3: The graph $\mathcal{G}$

Assume that $\mathcal{P}, \mathcal{Q}, \mathcal{R}, \mathcal{S}, \mathcal{T}$ and $\mathcal{U}$ are representable, say by $P, Q, R, S, T$ and $U$ respectively, and that there are no dependencies between processes where there is no $\rightarrow$ or - edge in 

Second, an arc between the vertices exists iff the vertices are dependent.

Third, the $\mathcal{P}_{i}, \mathcal{Q}_{j}$ can be decomposed as $\mathcal{P}_{i}=\mathcal{R}_{i 0} \otimes \mathcal{R}_{i 1}$ and $\mathcal{Q}_{j}=\mathcal{R}_{0 j} \oplus \mathcal{R}_{1 j}$. By the way, this together with $\mathcal{P}_{0} \oplus \mathcal{P}_{1}=\mathcal{Q}_{0} \otimes \mathcal{Q}_{1}$ gives:

$\left(\mathcal{R}_{00} \otimes \mathcal{R}_{01}\right) \oplus\left(\mathcal{R}_{10} \otimes \mathcal{R}_{11}\right)=\left(\mathcal{R}_{00} \oplus \mathcal{R}_{10}\right) \otimes\left(\mathcal{R}_{01} \oplus \mathcal{R}_{11}\right)$.

Fourth, if $R_{i j}$ are terms that denote the $\mathcal{R}_{i j}$, then:

$\left(R_{00} \otimes R_{01}\right) \oplus\left(R_{10} \otimes R_{11}\right) \stackrel{a x}{=}\left(R_{00} \oplus R_{10}\right) \otimes\left(R_{01} \oplus R_{11}\right)$.

Proof.

The first three claims are immediate by the definition of the graph operations $\|$ and $\bullet$, and the wel-formedness constraints. For the fourth claim we distinguish between the four choices for $\oplus, \otimes$ :

Case $\oplus, \otimes=\|$,$\| . Associativity and commutativity of \|$ suffice; these are expressed by axiom (A1).

Case $\oplus, \otimes=\|, \bullet$ or $\bullet, \|$. Since $R_{i j}$ denotes $\mathcal{R}_{i j}$, we conclude from the definition of $\mathcal{R}_{i j}$ and the first part of the lemma that the independence condition of the CCL law is satisfied. So one application of axiom (A2) does the job.

Case $\oplus, \otimes=\bullet, \bullet$. Since $R_{i j}$ denotes $\mathcal{R}_{i j}$, we conclude from the definition of $\mathcal{R}_{i j}$ and the first part of the lemma that $R_{01}$ and $R_{10}$ are independent. Now associativity of • (expressed by axiom (A1)) and the Independence Law (derivable from the axioms $(\mathbf{A 1}, \mathbf{A} 2))$ suffice.

Lemma 3.13 Term Existence

Let $P$ be a term, and $\mathcal{P}$ its graph. Let $\oplus$ be parallel or layered composition.

1. Suppose that $\mathcal{P}=\mathcal{S k i p}$ or $\mathcal{P}=\mathcal{X}$. Then $P \stackrel{a x}{=}$ skip or $P \stackrel{a x}{=} X$, respectively.

2. Suppose there exist graphs $\mathcal{P}_{i}$ such that $\mathcal{P}=\mathcal{P}_{0} \oplus \mathcal{P}_{1}$. Then there exist terms $P_{i}$ denoting $\mathcal{P}_{i}$ such that $P \stackrel{a x}{=} P_{0} \oplus P_{1}$.

Proof.

By induction on the structure of $P$. Part 1 is almost trivial. We apply case analysis on the syntactic form of $P$ :

Case $P \stackrel{\text { def }}{=}$ skip. Then apparently $\mathcal{P}=$ Skip, and trivially $P \stackrel{a x}{=}$ skip by reflexivity of axiomatic equality.

Case $P \stackrel{\text { def }}{=} Y$. Then apparently $X \stackrel{\text { def }}{=} Y$ and $\mathcal{P}=\mathcal{X}$, and again trivially $P \stackrel{a x}{=} X$. 
Case $P \stackrel{\text { def }}{=} Q_{0} \oplus Q_{1}$, where $\oplus$ is either $\|$ or $\bullet$. Now one of $Q_{j}$, say $Q_{0}$, denotes $\mathcal{S} k i p$, and the other one, $Q_{1}$, denotes $\mathcal{P}$. By induction $Q_{0} \stackrel{a x}{=}$ skip, and $Q_{1} \stackrel{a x}{=}$ skip or $\stackrel{a x}{=} X$. So by neutrality of skip for $\oplus$ (expressed by (A1)) we have either:

$$
\begin{array}{lllllll}
P & \stackrel{\text { def }}{=} & Q_{0} \oplus Q_{1} & \stackrel{\text { ax }}{=} & \text { skip } \oplus \text { skip } & \stackrel{a x}{=} \text { skip, } & \text { or: } \\
P & \stackrel{\text { def }}{=} & Q_{0} \oplus Q_{1} & \stackrel{a x}{=} & \text { skip } \oplus X & \stackrel{a x}{=} X .
\end{array}
$$

The proof of Part 2 is more interesting. Again, we apply case analysis on the syntactic form of $P$ :

Case $P \stackrel{\text { def }}{=}$ skip or $P \stackrel{\text { def }}{=} X$. Then at least one of $\mathcal{P}_{i}$, say $\mathcal{P}_{0}$, is Skip, and the other, $\mathcal{P}_{1}$, is $\mathcal{P}$. So take $P_{0} \stackrel{\text { def }}{=}$ skip and $P_{1} \stackrel{\text { def }}{=} P$. Then $P_{i}$ denotes $\mathcal{P}_{i}$, and by neutrality of skip for $\oplus$ we have:

$$
P \quad \stackrel{a x}{=} \quad \operatorname{skip} \oplus P \quad \stackrel{a x}{=} \quad P_{0} \oplus P_{1} .
$$

Case $P \stackrel{\text { def }}{=} Q_{0} \otimes Q_{1}$ where $\otimes$ is one of $\|$ or $\bullet$. Let $\mathcal{Q}_{j}$ be the graph denoted by $Q_{j}$. Put $\mathcal{R}_{i j}=\mathcal{P}_{i} \cap \mathcal{Q}_{j}$. By the Decomposition Lemma 3.12 we have:

$$
\begin{array}{ll}
(\mathbf{a} 0) & \mathcal{P}_{i}=\mathcal{R}_{i 0} \otimes \mathcal{R}_{i 1} \\
(\text { a1 }) & \mathcal{Q}_{j}=\mathcal{R}_{0 j} \oplus \mathcal{R}_{1 j} \\
(\mathbf{a 2}) & \left(R_{00} \oplus R_{10}\right) \otimes\left(R_{01} \oplus R_{11}\right) \stackrel{a x}{=}\left(R_{00} \otimes R_{01}\right) \oplus\left(R_{10} \otimes R_{11}\right),
\end{array}
$$

for all terms $R_{i j}$ that happen to denote the graphs $\mathcal{R}_{i j}$. By induction (applied to terms $Q_{j}$ with graph decomposition (a1)) there exist:

(b) terms $R_{0 j}, R_{1 j}$ denoting $\mathcal{R}_{0 j}, \mathcal{R}_{1 j}$, such that

(c) $\quad Q_{j} \stackrel{a x}{=} \quad R_{0 j} \oplus R_{1 j}$.

Now define the required terms $P_{i}$ by:

$$
\text { (d) } \quad P_{i} \stackrel{\text { def }}{=} \quad R_{i 0} \otimes R_{i 1} \text {. }
$$

It remains to verify the two claims about the $P_{i}$. First, term $P_{i}$ denotes $\mathcal{P}_{i}$ :

$$
\begin{array}{ll}
\text { def } & P_{i} \\
& \left\{(\mathrm{~d}): \text { definition } P_{i}\right\} \\
& R_{i 0} \otimes R_{i 1} \\
= & \left\{(\mathrm{b}): \text { definition } R_{i j}\right\} \\
& \mathcal{R}_{i 0} \otimes \mathcal{R}_{i 1} \\
= & \{(\mathrm{a} 0): \text { Decomposition Lemma } 3.12\} \\
& \mathcal{P}_{i}
\end{array}
$$

Second, the equality $P=P_{0} \oplus P_{1}$ is axiomatically provable:

$$
\begin{array}{ll}
\stackrel{P}{\text { def }} & \multicolumn{2}{l}{\text { (case assumption }\}} \\
& Q_{0} \otimes Q_{1} \\
\stackrel{a x}{=} & \{(\mathrm{c}): \text { induction }\}
\end{array}
$$




$$
\begin{array}{ll} 
& \left(R_{00} \oplus R_{10}\right) \otimes\left(R_{01} \oplus R_{11}\right) \\
\stackrel{\text { ax }}{=} & \{(\mathrm{b}, \mathrm{a} 2): \text { Decomposition Lemma } 3.12\} \\
& \left(R_{00} \otimes R_{01}\right) \oplus\left(R_{10} \otimes R_{11}\right) \\
\stackrel{\text { def }}{=} & \left\{(\mathrm{e}): \text { definition } P_{i}\right\} \\
& P_{0} \oplus P_{1} .
\end{array}
$$

This completes the proof of Part 2, hence of the entire Term Existence Lemma.

One may notice that precisely all axioms in (A1), (A2) have been used in the proofs of the two lemmas. Now we can state and proof the main result.

\section{Theorem 3.14 Modular Completeness}

For arbitrary terms $P, Q$ we have: $P=Q \quad \Longrightarrow \quad P \stackrel{a x}{=} Q$.

As a corollary we obtain the following result which was first proven by Gischer [Gis84] in his thesis.

\section{Corollary 3.15}

Let $\leadsto=\mathcal{A c t}^{2}$, i.e. all process variables are dependent, then

$$
P=Q \quad \Longrightarrow \quad P \stackrel{a x}{=} Q,
$$

where in proving $P \stackrel{a x}{=} Q$ we only need the axioms (A1).

Proof of the Completeness Theorem.

By induction on the size of the smaller one of $P, Q$; the size of a term $P$, denoted $|P|$, is the number of occurrences of skip, process variables, and parallel and layer operation symbols in $P$.

Without loss of generality assume that $|P| \leq|Q|$. We apply case analysis on the syntactic form of $P$ :

Case $P \stackrel{\text { def }}{=}$ skip or $P \stackrel{\text { def }}{=} X$. Then $Q$ denotes Skip or $\mathcal{X}$, respectively. So by the Term Existence Lemma: $Q \stackrel{a x}{=}$ skip $\stackrel{\text { def }}{=} P$ or $Q \stackrel{a x}{=} X \stackrel{\text { def }}{=} P$, respectively.

Case $P \stackrel{\text { def }}{=} P_{0} \oplus P_{1}$ where $\oplus$ is one of $\|$ or $\bullet$. Take $\mathcal{Q}_{j}$ to be the graph denoted by $P_{j}$. So by the Term Existence Lemma, reading $Q, \mathcal{Q}, j$ instead of $P, \mathcal{P}, i$, there exist terms $Q_{j}$ such that:

$$
\begin{aligned}
& \text { (a) } Q_{j} \text { denotes } \mathcal{Q}_{j} \text {, hence } Q_{j}=P_{j} \text { (semantically), } \\
& \text { (b) } Q \stackrel{a x}{=} Q_{0} \oplus Q_{1} .
\end{aligned}
$$

Observe that:

$$
\begin{aligned}
& \left|P_{i}\right|<\left|P_{0}\right|+1+\left|P_{1}\right|=\left|P_{0} \oplus P_{1}\right|=|P|, \quad \text { so } \\
& \min \left(\left|P_{i}\right|,\left|Q_{i}\right|\right) \leq\left|P_{i}\right|<|P|=\min (|P|,|Q|),
\end{aligned}
$$

hence, by induction applied to (a):

$$
\text { (c) } \quad P_{i} \stackrel{a x}{=} \quad Q_{i} \text {. }
$$

Now, by the case assumption, (c), and (b):

$$
P \quad \stackrel{\text { def }}{=} \quad P_{0} \oplus P_{1} \quad \stackrel{a x}{=} \quad Q_{0} \oplus Q_{1} \quad \stackrel{a x}{=} Q .
$$


This completes the proof of the Completeness Theorem.

Remark. Consider the process term

$$
(P \bullet Q) \|(R \bullet S)
$$

with $P \sharp S$ and $Q \sharp R$. Then none of the axioms in (A1) and neither (CCL-L) and (CCL-R) are applicable to this term. Hence the CCL law can not be derived from these laws.

Moreover a case analysis shows that every law of the form

$$
P \oplus(Q \otimes R)=P \otimes(Q \oplus R)
$$

can be derived from the axioms (A1) or is equal to (CCL-L) or (CCL-R).

Hence the CCL rule cannot be derived from simpler laws. (End of remark)

\section{References}

[CG88] C. Chou and E. Gafni. Understanding and verifying distributed algorithms using stratified decomposition. In Proc. 7th ACM Symposium on Principles of Distributed Computing. ACM, 1988.

[EF82] Elrad and N. Francez. Decomposition of distributed programs into communication closed layers. Science of Computer Programming, 2, 1982.

[GHS83] R.T. Gallager, P.A. Humblet, and P.M. Spira. A distributed algorithm for minimum-weight spanning trees. ACM TOPLAS, 5(1):66-77, Jan 1983.

[Gis84] J. L. Gischer. Partial Orders and the Axiomatic Theory of Shuffle. PhD thesis, Stanford University, 1984.

[JPSZ91] W. Janssen, M. Poel, K. Sikkel, and J. Zwiers. The primordial soup algorithm: A systematic approach to the specification and design of parallel parsers. In Proc. Computing Science in the Netherlands, pages 298-314, 1991.

[JPZ91] W. Janssen, M. Poel, and J. Zwiers. Action systems and action refinement in the development of parallel systems. In Proc. of CONCUR '91, pages 298-316. Springer-Verlag, LNCS 527, 1991.

[JPZ93] W. Janssen, M. Poel, and J. Zwiers. Action systems and action refinement in the development of parallel systems. Technical Report 93-14, University of Twente, 1993.

[JZ92a] W. Janssen and J. Zwiers. From sequential layers to distributed processes, deriving a distributed minimum weight spanning tree algorithm, (extended abstract). In Proc. 11th ACM Symposium on Principles of Distributed Computing, pages 215-227. ACM, 1992.

[JZ92b] W. Janssen and J. Zwiers. Protocol design by layered decomposition, a compositional approach. In J.Vytopil, editor, Proc. Formal Techniques in Real-Time and Fault-Tolerant Systems, pages 307-326. Springer-Verlag, LNCS 571, 1992. 
[KP87] S. Katz and D. Peled. Interleaving set temporal logic. In Proc. of the 6th ACM Symposium on Principles of Distributed Computing, Vancouver, pages 178-190, 1987.

[KP89] S. Katz and D. Peled. An efficient verification method for parallel and distributed programs. In Proc. of the REX workshop on Linear Time, Branching Time and Partial order in Logics and Models for Concurrency, Noordwijkerhout, Springer LNCS 354, pages 489-507. Springer-Verlag, 1989.

[KP90] S. Katz and D. Peled. Interleaving set temporal logic. Theoretical Computer Science, 75(2), 1990.

[KP92] S. Katz and D. Peled. Verification of distributed programs using representative interleaving sequences. Distributed Computing, 6(2), 1992.

[Maz89] A. Mazurkiewicz. Basic notions of trace theory. In J.W. de Bakker, W.-P. de Roever, and G. Rozenberg, editors, Proc. of the REX workshop on Linear Time, Branching Time and Partial order in Logics and Models for Concurrency, Noordwijkerhout 1988, Springer LNCS 354, pages 285-363, 1989.

[Mil80] R. Milner. A Calculus of Communicating Systems, volume 92 of Lecture Notes in Computer Science. Springer-Verlag, 1980.

[PZ92] M. Poel and J. Zwiers. Layering techniques for development of parallel systems. In Proc. $C A V, 1992$.

[PZ93a] M. Poel and J. Zwiers. Closed layers in the presence of conspiracy and cascading. 1993.

[PZ93b] M. Poel and J. Zwiers. Modular completeness for communication closed layers. Technical report, University of Twente, 1993.

[SdR89] F.A. Stomp and W.P. de Roever. Designing distributed algorithms by means of formal sequentially phased reasoning. In J.-C. Bermond and M. Raynal, editors, Proc. of the 3rd International Workshop on Distributed Algorithms, Nice, LNCS 392, pages 242-253. Springer-Verlag, 1989.

[VTL82] J. Valdes, R.E. Tarjan, and E.L. Lawler. The recognition of series parallel digraphs. SIAM Journal of Computing, 2(11):298-313, 1982.

[Zwi89] J. Zwiers. Compositionality, Concurrency and Partial Correctness. Springer LNCS 321, 1989.

[Zwi91] J. Zwiers. Layering and action refinement for timed systems. In Proc. of the REX Workshop on Real Time: Theory and Practice, Mook, the Netherlands, June 3-7 1991. Springer-Verlag. 